\title{
Effets de l'hydrogel sur la survie et la croissance des plantules d'arganier sur une parcelle de régénération en climat aride.
}

\author{
Chamchelmaarif Defaa (1-2), Ahmed Achour (1), Abdelhamid El Mousadik (2), Fouad Msanda (2) \\ (1) Direction Régionale des Eaux et Forêts et de la Lutte Contre la Désertification du Sud Ouest - Agadir (Morocco) ; \\ (2) Laboratoire de Biotechnologie et de Valorisation des Ressources Naturelles, Université lbn Zohr - Agadir (Morocco). \\ Corresponding author: Chamchelmaarif Defaa : ch.defaa@uiz.ac.ma
}

Original submitted in on 26 th June 2015. Published online at www.m.elewa.org on $31^{\text {st }}$ August 2015 http://dx.doi.org/10.4314/jab.v92i1.3

\section{RÉSUMÉ}

Objectifs: L'objet de cette étude est l'évaluation à court terme de l'opportunité de réduction de l'eau d'arrosage en amendant le sol par un hydrogel ainsi que l'étude de ses effets sur le taux de survie et les paramètres de croissance de jeunes plantules d'arganier dans une parcelle de régénération en climat aride.

Méthodologie et résultats : un dispositif expérimental complètement randomisé avec trois répétitions de 33 plants pour chaque traitement : sol amendé en hydrogel et sol témoin. Les plants sur sol témoin ont été arrosés mensuellement et les plants sur sol amendé une fois tous les deux mois. Le traitement n'a pas eu d'effet significatif sur le taux de survie, il a cependant amélioré significativement la croissance en hauteur, le diamètre au collet et l'indice d'élancement des plants sur sol amendé.

Conclusions et applications : L'amendement du sol en PSA, malgré la réduction de moitié de la fréquence d'arrosage, a eu un impact positif sur les paramètres de développement des plants d'arganier durant la première campagne tout en gardant des taux de survie similaires. Les résultats obtenus ont montré que l'amendement du sol en hydrogel a permis d'économiser l'eau d'arrosage, de réduire les effets du stress hydrique dans ce périmètre de régénération après la transplantation des plantules d'arganier.

Mots-clés: Hydrogel, régénération des écosystèmes, climat aride, stress hydrique, adaptation aux changements climatiques, Argania spinosa.

First-year effects of hydrogel on survival and growth of Argania spinosa seedlings in a forest restoration trial in arid climate.

\section{ABSTRACT}

Objectives: The purpose of this study is the short-term assessment of the appropriateness of reducing irrigation water by amending the soil with hydrogel and the study of its effects on survival and young seedling growth parameters in forest restoration in arid climate.

Methodology and results: A completely randomized experimental design with three replications of 33 plants for each treatment, soil amended hydrogel and control soil. The plants in control soil were watered monthly and plants in amended soil were watered only once every two months. The treatment had no significant effect on 

sur une parcelle de régénération en climat aride.

the survival, however, it significantly improved height growth, root collar diameter and the slenderness index of plants on soil amended.

Conclusions and applications: Hydrogel amendment of soil, despite halving the frequency of watering, had a positive impact on the development of argan seedling's parameters during this campaign while keeping similar survival rates. The results obtained showed that the amendment of the soil by hydrogel has saved the irrigation water, reduced the effects of water stress in this trial after transplantation of argan seedlings.

Keywords: Polyabsorbent polymer, forest restoration, arid climate, stress hydric, climate change mitigation, Argania spinosa.

\section{INTRODUCTION}

L'arganier (Argania spinosa (L.) Skeels), espèce emblématique et endémique du sud-ouest marocain, joue un rôle de première importance dans la vie socio-économique et culturelle de la population locale, vu qu'il s'agit d'un arbre forestier, fruitier, fourrager et produisant un bois très dur et du charbon de très haute qualité (Photo 1).Ces dernières décennies, ces forêts ont subi d'innombrables perturbations d'ordre climatique et anthropozoogène qui menacent la pérennité même de ces forêts en réduisant leurs densités et leurs aires de répartition. La FAO (2010) a estimé cette régression à $7400 \mathrm{Ha}$ /an entre 1995 et 2005 dans un contexte caractérisé par un manque presque total de toute régénération naturelle de cet écosystème forestier à cause du surpâturage et du ramassage systématique des noix d'argane. Depuis la fin des

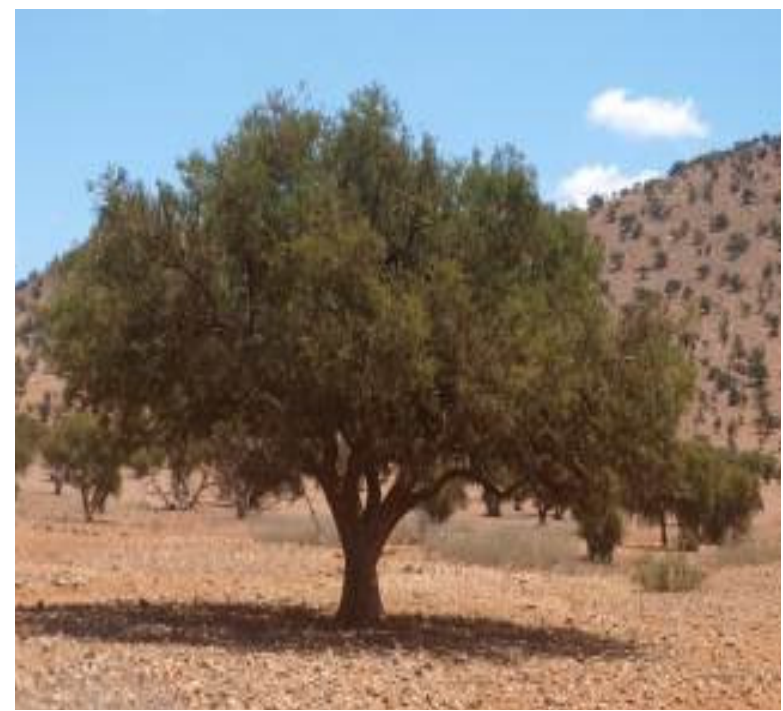

années 90, le HCEFLCD a lancé un vaste programme de restauration de ces écosystèmes basé sur la régénération assistée par plantation de l'arganier. Ces programmes n'ont, à ce jour, presque jamais atteints les résultats escomptés et les périmètres de régénération sont repris plusieurs fois de suite pour atteindre un taux de réussite satisfaisant, engendrant ainsi d'une part des coûts supplémentaires et d'autre part, l'allongement de la période de mise en défens ce qui prive les éleveurs d'un espace vital pour leur bétail, constituant ainsi une source de tension permanente entre ces usagers et l'administration forestière. Parmi les causes de ces échecs figure le climat caractérisé par des températures extrêmes et une irrégularité des précipitations ainsi que la rareté des points d'eau pour l'arrosage surtout pendant la période estivale.

Photo 1 : Argania spinosa ; arbre adulte (à gauche) et fruits mûrs (à droite).

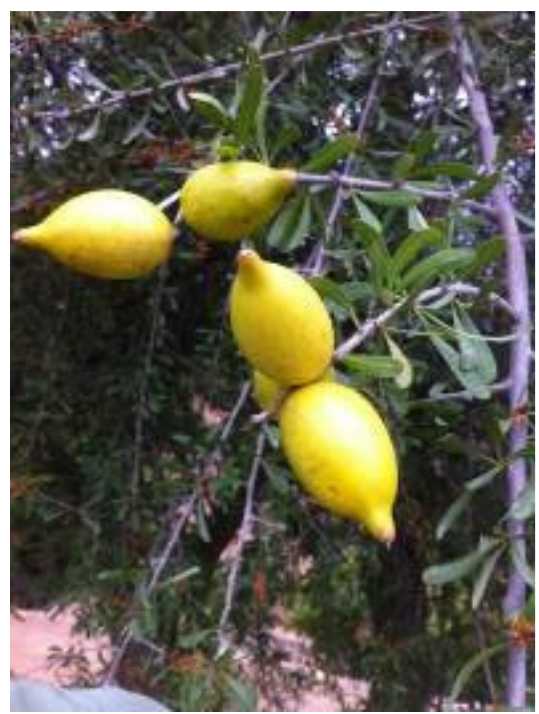


Les hydrogels sont des polymères organiques superabsorbants (PSA) en mesure de retenir des dizaines de fois leur poids en eau courante (Photo 2). Ghebru et al. (2007) a étudié deux PSA

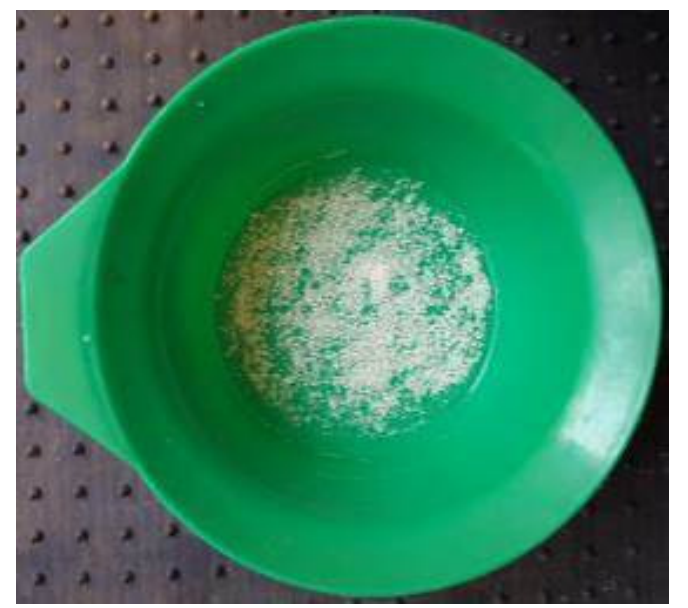

Photo 2 : PSA ; sec (à gauche) ; pré-hydraté (à droite).

Ce réservoir d'eau supplémentaire pour le système sol-plante contribue à la diminution du stress hydrique par l'augmentation de l'eau mise à la disponibilité de la plante dans le sol (Agaba et al. 2010, Goeppel 2014). Cette réserve peut être facilement transférée à l'arbre (Hüttermann et al. 1999) mitigeant ainsi les effets de sècheresse. L'utilisation d'hydrogels dans les plantations forestières peut être considérée comme une méthode pour réduire le stress du matériel transplanté avant la formation de nouvelles racines ou jusqu'à ce que la pluie fournisse l'humidité nécessaire aux plantules (Thomas et Heagney 2011). La survie des arbres étant critique dans les 2 premières années, l'application d'hydrogel pourrait contribuer à la réussite de plantation d'arbres à grande échelle (Agaba et al. 2010). Parvanak et

\section{MATÉRIEL ET MÉTHODES}

Zone d'étude : L'expérimentation a été menée dans la forêt domaniale d'Idaouzal (Province de Taroudant) située à moins d'un $\mathrm{Km}$ au nord de la localité d'Argana $\left(9^{\circ} 08^{\prime} 00^{\prime \prime} \mathrm{W}, 30^{\circ} 46^{\prime} 59^{\prime \prime} \mathrm{N}\right.$, altitude $\left.805 \mathrm{~m}\right)$. La zone d'étude, clôturée, est située à l'intérieur d'un grand périmètre de régénération assistée d'arganier de $314 \mathrm{Ha}$. commerciaux et a conclu que Stockosorb $₫$ est celui qui absorbe le plus d'eau et qu'il peut absorber jusqu'à 300 fois son poids en solution à pH neutre (Silva et al. 2012).

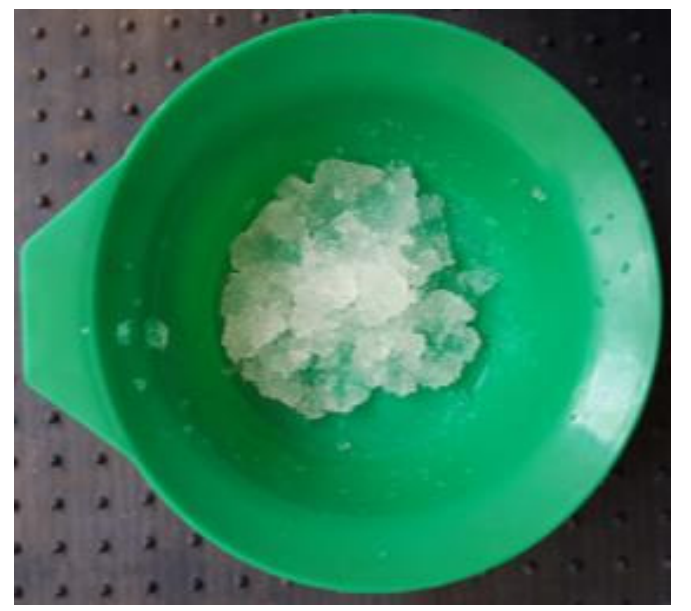

Chamheidar (2014) ont montré que l'utilisation d'un PSA permet de réduire de moitié la fréquence d'arrosage tout en gardant un enracinement optimal chez une rosacée. D'autres études ont montré que la confection d'une couche de Stockosorb ${ }^{\circledR}$ en profondeur dans le sol permettait non seulement de gonfler et de retenir l'eau de pluie (Wei et al. 2014), mais constitue une technique efficace pour créer de larges « réservoirs » provisoires d'eau au niveau des horizons supérieurs (Wei et Durian 2014). Le principal objet de cette étude est l'évaluation à court terme de l'opportunité de réduire de moitié la fréquence d'arrosage en amendant le sol par un PSA, ainsi que l'étude des effets de cet amendement sur le taux de survie et les paramètres de croissance de jeunes plantules d'arganier en plein champs.

Le bioclimat est du type aride à hiver frais avec des précipitations moyennes annuelles de $216,6 \mathrm{~mm}$ et une température moyenne de $19^{\circ} \mathrm{C}$. La zone est connue pour ses fortes chaleurs estivales (la moyenne des maxima du mois le plus chaud est de $41,8^{\circ} \mathrm{C}$ ) ainsi qu'une amplitude thermique annuelle de $22^{\circ} \mathrm{C}$. Des échantillons de sol ont 


\section{Defaa et al. J. Appl. Biosci. Effets de l'hydrogel sur la survie et la croissance des plantules d'arganier}

sur une parcelle de régénération en climat aride.

été prélevés à l'aide d'une tarière à des profondeurs de 0$20 \mathrm{~cm}$ et $20-40 \mathrm{~cm}$. Ces échantillons ont été analysés par la méthode standard pour déterminer leur granulométrie Tab. 1: Cactéristiques physico-chimiques du sol étudié.

\begin{tabular}{lllllll}
\hline Profondeur du sol $(\mathbf{c m})$ & $\% \mathbf{C}$ & $\% \mathbf{N}$ & $\mathbf{C} / \mathbf{N}$ & $\%$ MO & pH & $\mathbf{P}$ \\
\hline $0-20$ & 2,90 & 1,07 & 27,08 & 5,01 & 7,16 & 131,55 \\
\hline $20-40$ & 1,12 & 0,34 & 32,94 & 1,94 & 7,66 & 71,69 \\
\hline
\end{tabular}

$(\% \mathrm{C})$ : carbone organique total ; $(\% \mathrm{~N})$ : azote total ; $(\mathrm{C} / \mathrm{N})$ : rapport $\mathrm{C} / \mathrm{N}$; $(\% \mathrm{MO})$ : matière organique ; $(\mathrm{pH})$ : $\mathrm{pH}$ du sol mesuré dans une solution acqueuse $1: 5 ;(\mathrm{P})$ : phosphore disponible en ppm.

Matériel utilisé : Au total, 198 plants d'arganier ont été utilisés. Ce lot est issu de gaines ayant la même origine, à savoir l'arganeraie de la région des Idaoumoumen (Province de Taroudant). Les plantules ont été élevées dans la pépinière forestière d'Oulad Taima située à 20 $\mathrm{Km}$ de la zone d'étude. Ladite pépinière, qui produit plusieurs centaines de milliers de plants d'arganier par an, est le principal fournisseur pour les périmètres de régénération d'arganier dans la région. A leur mise en terre les plants étaient âgés de 9 mois. Le PSA utilisé est le Stockosorb 500 sous forme granulée. Cet hydrogel hydrophile est constitué de copolymères d'acrylates de potassium et de polyacrylamides réticulés (Shi et al. 2010).

Dispositif expérimental : La parcelle expérimentale a été préparée pour accueillir un dispositif expérimental complètement randomisé avec 3 répétitions et 2 traitements : sol amendé en PSA et dosé à $0,1 \%(\mathrm{w} / \mathrm{w})$ et le témoin (sol sans amendement). L'unité expérimentale étant constituée de 33 plants d'arganier. Pendant toute la durée de l'étude, les variables mesurées sont la hauteur et le diamètre au collet de chaque plant.

Préparation du sol et plantation : La parcelle a été tracée selon une maille de 8 sur 5 mètres pour la préparation du sol en vue de la mise en terre des plants d'arganiers. Des potets simples ont été ouverts avec des dimensions cubiques de 0,60 m. En ce qui concerne le sol amendé, 1/3 du PSA sous forme de gel pré-hydraté a

\section{RÉSULTATS ET DISCUSSIONS}

Survie des jeunes plantules d'arganier: La fig.1 présente l'évolution des taux de survie des plants d'arganiers enregistrés au cours de la période d'étude. Quoique statistiquement non significatif ( $P=0,080)$, un gain de $8,08 \%$ sur le taux de survie a été observé pour les plants en sol amendé par rapport au témoin. Certains travaux ont conclu que l'application du PSA n'a eu aucun effet sur la survie de plusieurs essences forestières en et leurs cactéristiques physico-chimiques. Le sol de la zone d'étude est un sol à texture argileuse, relativement riche en matière organique avec un pH neutre et (Tab. 1). été déposé en couche au fond du trou de plantation et les 2/3 restants ont été bien mélangés à la terre avec laquelle le trou a été rebouché. Un impluvium en croissant avec un bourrelet de $30 \mathrm{~cm}$ de haut et 1 mètre de rayon a été confectionné en aval du plant. Le plant a été ensuite mis en terre et le sol amendé a été recouvert d'une couche de terre sans PSA sur environ $5 \mathrm{~cm}$. La terre autour des plants a été tassée et le premier arrosage a suivi immédiatement. La mise en terre des plants a été effectuée le 01 janvier 2011.

Arrosages : Un arrosage régulier mensuel de 40 litres d'eau/plant a été assuré pour les plants témoins. Un arrosage bimensuel de 40 litres d'eau/plant a été assuré aux plants au niveau du sol amendé par le PSA.

Analyse statistique : Les effets des deux traitements (sol amendé et témoin) sur les variables quantitatives mesurées ont subi une analyse de la covariance en considérant les valeurs initiales de chaque variable mesurée comme des covariables. Auparavant, les données relatives à la survie des plants ont subi une transformation angulaire du type arc-sinus de la racine carrée $\left(Y=2 a r \sin X^{1 / 2}\right)$, les données brutes étant exprimées en valeurs de 0 à 1 (Dagnelie 2011). Les données ont subi des transformations logarithmiques pour satisfaire aux besoins d'une analyse de la covariance. Les données ont été traitées par le logiciel statistique SPSS version 19.

climat tempéré (Sarvas, 2003 ; Böhlenius et Övergaard, 2014). D'autres études, ont montré que l'amendement du sol en PSA a permis de prolonger la survie des plantules de 9 essences forestières sur différents de sols et dans des conditions de stress hydrique (Agaba et al., 2010). Plusieurs auteurs ont montré que l'incorporation du PSA au sol a eu un impact positif sur le taux de survie des 
jeunes arbres : Sarvas et al. (2007), Thomas et Heagney (2011).

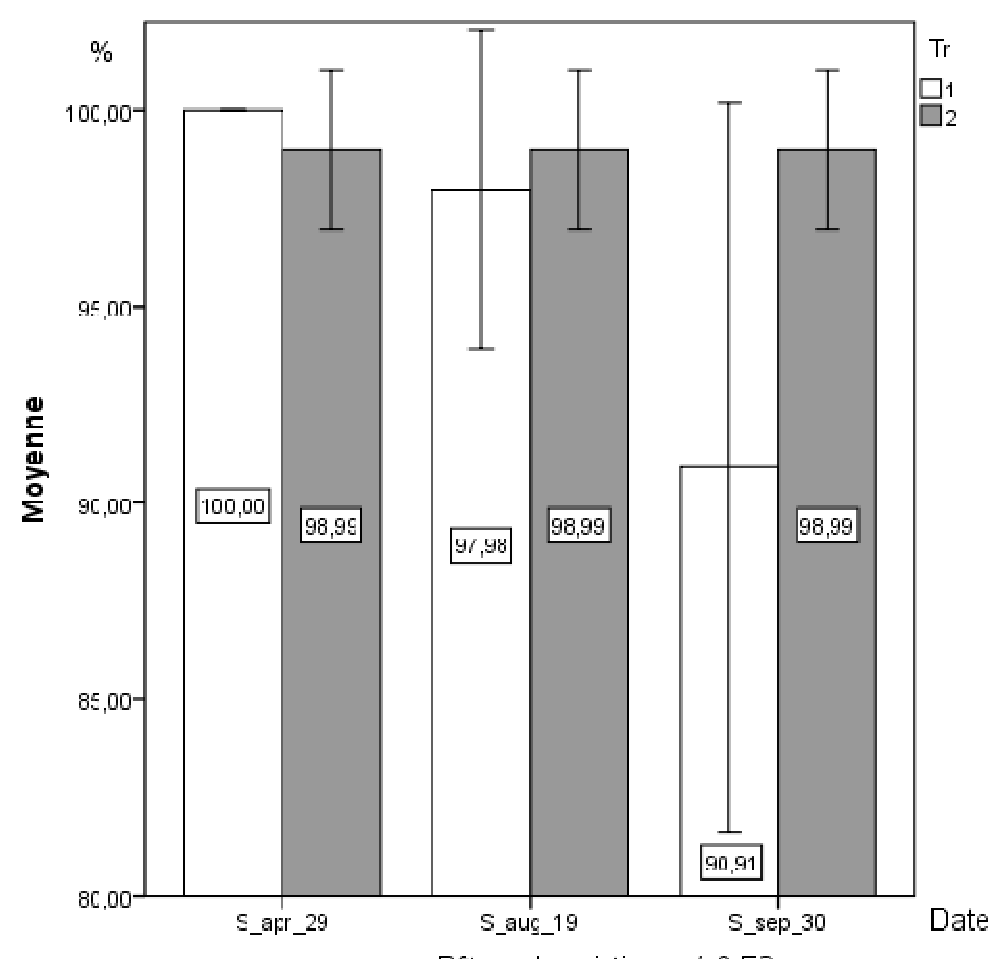

Fig. 1 : Taux de survie enregistrés. ( $\operatorname{Tr} 1$ ): Témoin ; $(\operatorname{Tr} 2)$ : sol amendé avec le PSA; (S_apr_29): taux de survie observé le 29 avril 2011; (S_aug_19): taux de survie observé le 19 aout 2011; (S_sep_30): taux de survie observé le 30 septembre 2011.

Hüttermann et al.(1999), Arbona et al.(2005), Beniwal et al.(2011) et Fajardo et al.(2013) ont montré des effets significatifs de l'amendement du sol en PSA sur l'amélioration de l'humidité du sol, ainsi que sur les taux de survie et la croissance de plusieurs espèces. L'absence de différence significative entre les taux de survies observés pour les deux traitements (sol amnedé et sol témoin) est un résultat très positif en soit vu que les plants sur sol amendé n'ont été arrosés qu'une fois sur deux, permettant de garder un taux de survie similaire tout en économisant $50 \%$ d'eau.

Croissance en hauteur des plants : Les résultats ont montré des effets très hautement significatifs de l'amendement du sol en PSA sur la croissance en hauteur des plants d'arganiers et ce dès le quatrième mois après la plantation (Tab. 2).

Tab. 2 : Effets de l'amendement du sol en PSA sur la croissance en hauteur.

\begin{tabular}{llll}
\hline Date & ddl & F & P \\
\hline Avril 2011 & 1 & 44,42 & $<0,001$ \\
\hline Aout 2011 & 1 & 61,86 & $<0,001$ \\
\hline Septembre 2011 & 1 & 53,08 & $<0,001$ \\
\hline
\end{tabular}




\section{Defaa et al. J. Appl. Biosci. Effets de l'hydrogel sur la survie et la croissance des plantules d'arganier sur une parcelle de régénération en climat aride.}

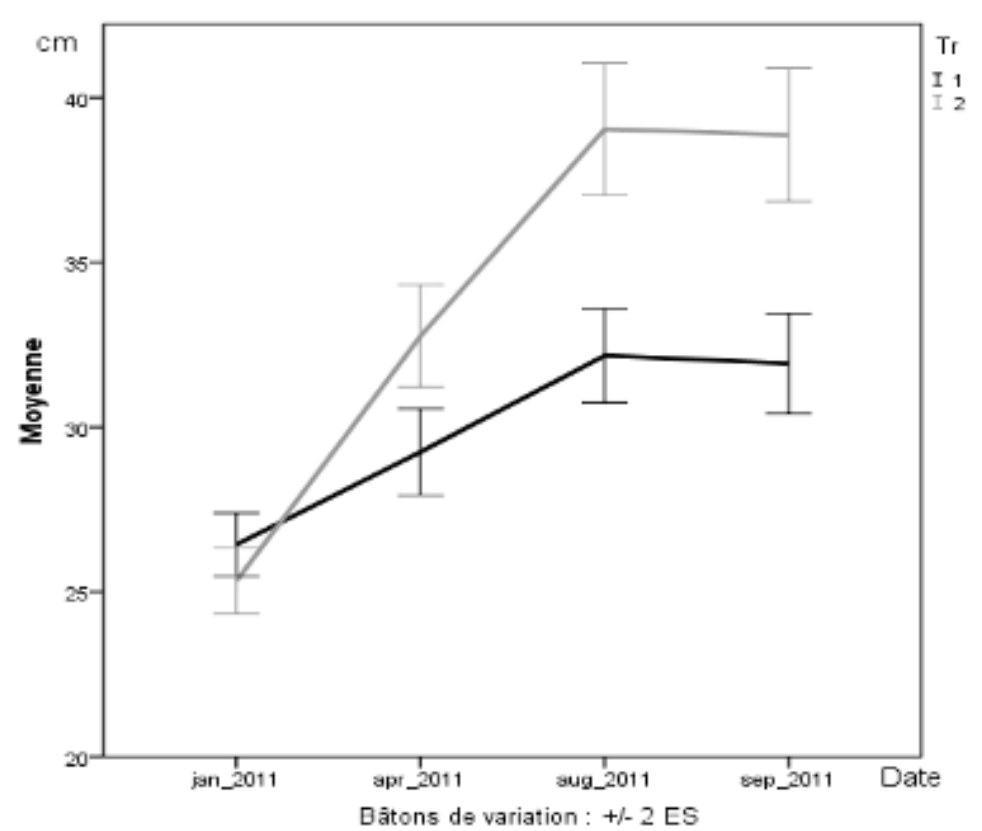

Fig. 2: Hauteurs moyennes des plants. ( $\operatorname{Tr} 1$ ): témoin ; $\operatorname{Tr} 2$ ): sol amendé avec le PSA; (jan_29): hauteur observée le 01 janvier 2011 ; (apr_29): hauteur observée le 29 avril 2011; (aug_19): hauteur observée le 19 aout 2011; (sep_30): hauteur observée le 30 septembre 2011.

L'amendement du sol en PSA s'est traduit par une amélioration très hautement significative de la croissance en hauteur des plants d'arganiers dès la saison printanière (Fig. 2). Si certains travaux (Swietlik 1989, Böhlenius et Övergaard 2014) n'ont montré aucun effet de l'amendement du sol en PSA sur la croissance en hauteur des plants, de nombreux travaux ont signalé des effets positifs (Hüttermann et al.,1999; Arbona et al., 2005; Beniwal et al., 2011, Silva et al. 2012, Fajardo et al., 2013). Chen et al. (2004), qui a travaillé sur un sol salin, a montré que l'incorporation du Stockosorb au sol a permis de multiplier par 2,7 la biomasse aérienne de plants de peupliers élevés sous serre et ce malgré la salinité du sol. II a aussi montré que les plants élevés dans un sol amendé avaient des racines 3,5 fois plus longues que celles élevées dans le sol non amendé. L'amendement du sol en PSA, grâce à ses mini réservoirs d'eau, a permis d'atténuer les effets du stress hydrique en continuant à fournir aux racines l'eau nécessaire à la survie et au développement de la plante malgré le déficit hydrique engendré par la réduction de la fréquence d'arrosage. Des résultats similaires ont été obtenus par Shi et al. (2010) qui a montré que l'utilisation du Stockosorb a permis de reporter de 31 jours l'apparition de blessures foliaires dues au stress hydrique et à la salinité. II a conclu qu'en condition de sècheresse, le PSA a permis d'améliorer la consommation d'eau chez le peuplier, d'augmenter les échanges gazeux foliaires, la matière sèche racinaire et la photosynthèse nette chez la plante.

Diamètre au collet : Les résultats obtenus montrent un effet très hautement significatif de l'amendement du sol en SAP sur le diamètre au collet des plants d'arganiers en fin de saison (Tab. 3).

Tab. 3 : Effets du traitement sur le diamètre au collet.

\begin{tabular}{llll}
\hline Date & Ddl & F & P \\
\hline Septembre 2011 & 1 & 68,04 & $<0,001$
\end{tabular}

Les valeurs moyennes du diamètre basal des jeunes arganiers plantés en sol amendé (Tab. 4) montrent un gain de $27 \%$ par rapport aux témoins après une période de neuf mois. 

sur une parcelle de régénération en climat aride.

Tab. 4 : Diamètre au collet moyen $(\mathrm{mm})$ des plants d'arganier 9 mois après la plantation.

\begin{tabular}{lll}
\hline Traitement & Moyenne & Erreur standard \\
\hline Témoin & 4,56 & 0,129 \\
\hline Sol amendé en SAP & 5,79 & 0,144 \\
\hline
\end{tabular}

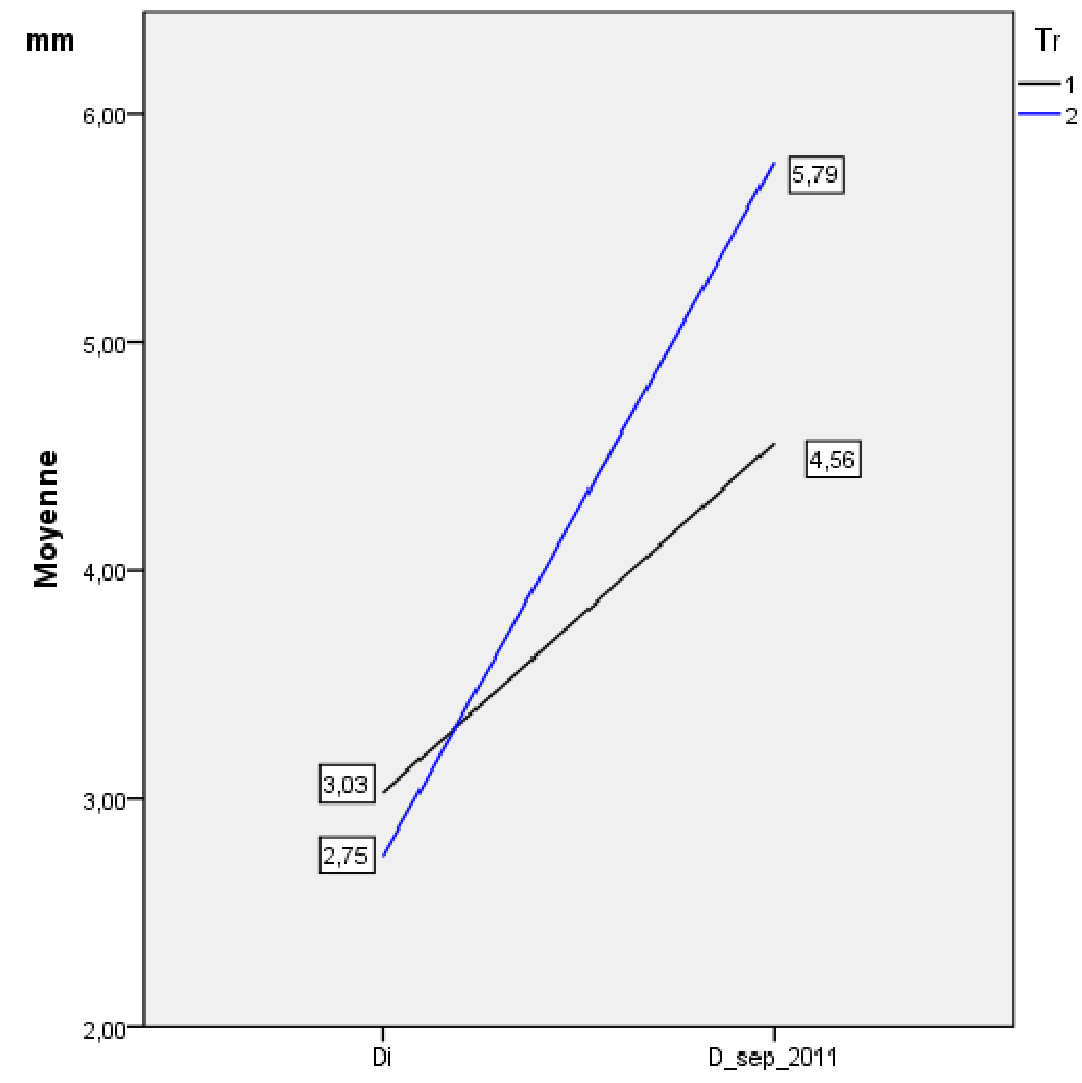

Fig. 3: Diamètre au collet moyen des plants d'arganier 9 mois après la plantation. $(\operatorname{Tr} 1)$ : control ; $(\operatorname{Tr} 2)$ : sol amendé avec le PSA ; (Di): Diamètre au collet moyen observé le 01 janvier 2011 ; (D-sep_2011): Diamètre au collet moyen observé le 30 septembre 2011.

Neuf mois après la plantation, l'amendement du sol en PSA a permis de multiplier par 2,1 fois le diamètre au collet moyen des arganiers par rapport à sa valeur initiale contre seulement 1,5 fois pour le témoin (Fig.3). Ces résultats confirment les tendances observées pour la croissance en hauteur décrites précédemment et sont conformes à ceux trouvés par Sarvas (2003) pour les plants d'Épicéa commun et Wróblewska et al. (2012) sur trois espèces ornementales pérennes. D'un autre côté, les travaux menés par Chirino et al. (2011) sur le chêneliège en climat méditerranéen n'ont montré aucun effet significatif de l'amendement du sol en Stockosorb sur le diamètre au collet des plants.

Élancement des plants : L'amendement du sol en SAP a eu un effet hautement significatif sur le rapport H/D qui traduit l'élancement du plant (Tab. 5).

Tab. 5: Effets du traitement sur l'élancement des plants.

\begin{tabular}{llll}
\hline Date & Ddl & F & P \\
\hline Septembre 2011 & 1 & 8,641 & 0,004 \\
\hline
\end{tabular}




\section{Defaa et al. J. Appl. Biosci. Effets de l'hydrogel sur la survie et la croissance des plantules d'arganier}

sur une parcelle de régénération en climat aride.

Plus cet indice est faible plus l'arbre est solide (De Champs 1987). Les moyennes présentées sur le Tab. 6 montrent que l'amendement du sol en PSA a permis de réduire le rapport $H / D$ et d'obtenir des plants plus vigoureux dès la première année.

Tab. 6: Élancement moyen des plants d'arganier 9 mois après la plantation.

\begin{tabular}{lll}
\hline Traitement & Moyenne & Erreur standard \\
\hline Témoin & 72,01 & 1,60 \\
\hline SAP & 68,17 & 1,33 \\
\hline
\end{tabular}

Les travaux menés par Chirino et al. (2011) sur le chêneliège en sol limoneux ont quant à eux conclu que

\section{CONCLUSION}

L'amendement du sol en PSA, malgré la réduction de moitié de la fréquence d'arrosage, a impacté positivement les paramètres de développement des plants d'arganier durant la première campagne tout en gardant des taux de survie similaires au témoin. Dans un contexte caractérisé par la rareté de la ressource hydrique induite par les changements climatiques, les résultats de la présente étude montrent que l'utilisation du SAP a permis d'économiser l'eau d'arrosage, de réduire les effets du stress hydrique après la transplantation des plantules

\section{REMERCIEMENTS}

Les auteurs remercient le Centre de Recherche Forestière pour avoir procédé aux analyses du sol, ainsi que toute l'équipe de la Direction Régionale des Eaux et Forêts et de la Lutte Contre la Désertification du Sud-

\section{RÉFÉRENCES}

Agaba H, Baguma Orikiriza LJ, Esegu O, Francis J, Obua J, Kabasa JD, Hüttermann A, 2010. Effects of hydrogel amendment to different soils on plant available water and survival of trees under drought conditions. Clean-Soil, Air, Water, 38(4): 328-335

Arbona V, Iglesias DJ, Jacas J, Primo-Millo E, Talon M, Gómez-Cadenas A, 2005. Hydrogel substrate amendment alleviates drought effects on young citrus plants. Plant and Soil 270: 73-82

Beniwal RS, Hooda MS, Polle A, 2011. Amelioration of planting stress by soil amendment with a hydrogel-mycorrhiza mixture for early establishment of beech (Fagus sylvatica L.) seedlings. Annals of Forest Science 68:803-810

Böhlenius H and Övergaard R, 2014. Effects of Direct Application of Fertilizers and Hydrogel on the l'amendement du sol en PSA a augmenté cet indice par rapport au témoin.

d'arganier. Elle pourrait être considérée comme un bon outil d'adaptation aux changements climatiques que connaissent le Maroc et la région du sud-ouest en particulier. La survie et la bonne reprise étant deux facteurs très importants pour la réussite des périmètres de régénération et la restauration des écosystèmes forestiers, l'amendement du sol en SAP semble être une technique prometteuse pour améliorer la réussite des opérations visant la restauration de ces écosystèmes en zone aride.

Ouest pour leur aimable assistance durant toutes les phases de cette recherche, incluant l'apport des plantules, la plantation et les différents travaux d'entretien.

Establishment of Poplar Cuttings. Forests 5 : 2967-2979

Chen S, Zommorodi M, Fritz E, Wang S, Hüttermann A, 2004. Hydrogel modified uptake of salt ions and calcium in Populus euphratica under saline conditions. Trees 18:175-183

Chirino E, Vilagrosa A, Vallejo VR, 2011. Using hydrogel and clay to improve the water status of seedlings for dryland restoration. Plant Soil 344: $99-110$

Dagnelie $P, 2011$. Statistique théorique et appliquée. Tome 2. Inférence statistique à une et à deux dimensions. De Boeck, Bruxelles, Belgium

De Champs J, 1987. Mesures sylvicoles préventives. Revue Forestière Française $4: 313-322$

Fajardo L, Rodríguez JP, González V, Briceño-Linares JM, 2013. Restoration of a degraded tropical dry 
forest in Macanao, Venezuela. Journal of Arid Environments 88: 236-243

Food and Agriculture Organization of the United Nations, 2010. Evaluation des ressources forestières mondiales 2010 Rapport national Maroc. http://www.fao.org/docrep/013/al574F/al574F.pd $f$

Ghebru MG, Du Toit ES, Steyn JM, 2007. Water and nutrient retention by Aquasoil $\odot$ and Stockosorb $\AA$ polymers. South African Journal of Plant and Soil 24(1): 32-36

Goeppel AE, 2014. Evaluating Jack Pine Seedling Characteristics in Response to Drought and Outplanting, Master of Science, Forest Biology and Management, Department of Renewable Resources, University of Alberta

Hüttermann A, Zommorodi M, Reise K, 1999. Addition of hydrogels to soil for prolonging the survival of Pinus halepensis seedlings subjected to drought. Soil \& Tillage Research $50: 295-304$

Parvanak K and Chamheidar H, 2014. The effect of polyacryl amide a 200 polymer on optimization of moisture and rooting indices of Rosa $\mathrm{sp}$. International Journal of Biosciences 5(6):119127

Sarvas M, 2003. Effect of desiccation on the root system of Norway spruce (Picea abies [L.] Karst.) seedlings and a possibility of using hydrogel STOCKOSORB $B$ for its protection. Journal of Forest Science 49(11): 531-536

Sarvaš $M$, Pavlenda $P$, Takáčová $E, 2007$. Effect of hydrogel application on survival and growth of pine seedlings in reclamations. Journal of Forest Science 53(5): 204-209

Shi Y, Li J, Shao J, Deng S, Wanga R, Li N, Sun J, Zhang $\mathrm{H}$, Zhu $\mathrm{H}$, Zhang $\mathrm{Y}$, Zheng $X$, Zhou D, Hüttermann A, Chen S, 2010. Effects of Stockosorb and Luquasorb polymers on salt and drought tolerance of Populus popularis. Scientia horticulturae, 124: 268-273

Silva DP, Pozza AA, Leite BS, Leite SA, 2012. Investigation of hydrogel application on the culture of beans to optimise water Resource and productivity. Chemical Engineering Transactions 29: $511-516$

Swietlik D, 1989. Effect of soil amendment with Viterra hydrogel on establishment of newly-planted grapefruit trees CV Ruby Red. Science and Plant Analysis. Communications in Soil Science and Plant Analysis, 20(15-16): 1697-1705
Thomas DS and Heagney GA, 2011. A root drench in unsaturated hydrogel solution reduced stress in transplanted Eucalyptus pilularis cuttings but was ineffective for seedlings: a glasshouse experiment. Australian Forestry 74(2): 133-140

Wei $Y$ and Durian DJ, 2014. Rain water transport and storage in a model sandy soil with hydrogel particle additives. The European Physical Journal E 37(97): 1-11

Wei Y, Cejas CM, Barrois R, Dreyfus R, Durian DJ, 2014. Morphology of Rain Water Channeling in Systematically Varied Model Sandy Soils. Physical Review Applied 2 (044004): 1-12

Wróblewska K, Debicz R, Babelewski P, 2012. The influence of water sorbing geocomposite and pine bark mulshing on growth and flowering of some perennial species. Acta Scientiarum Polonorum, Hortorum Cultus 11(2): 203-216. 